\title{
Application of virtual windows to determine the path of a uniformly moving obstacle
}

\author{
M.U. Kamaluddin, Hj. M.A. Hj. Mansor \\ Faculty of Electrical Engineering, MARA University of Technology, 40450 Shah Alam, Selangor, Malaysia
}

\begin{tabular}{l}
\hline \hline Article Info \\
\hline Article history: \\
Received Aug 14, 2018 \\
Revised Oct 15, 2018 \\
Accepted Oct 29, 2018 \\
\hline
\end{tabular}

\begin{abstract}
Two virtual windows are used to determine the path of a single uniformly moving obstacle. If the path of the obstacle crosses the two virtual windows, then its path can be easily determined. A simulation is implemented to ascertain the viability and accuracy of this technique.
\end{abstract}

\author{
Keywords: \\ Path determination \\ Uniformly moving obstacle \\ Virtual window
}

\section{Corresponding Author:}

M.U. Kamaluddin,

Faculty of Electrical Engineering,

MARA University of Technology,

40450 Shah Alam, Selangor, Malaysia.

Email: mohdu833@salam.uitm.edu.my

\section{INTRODUCTION}

In recent years, many researchers have their attention pointed to the solution of obstacle avoidance in path planning. This in part is due to the extension and interest in cruise control in land vehicles, unmanned vehicle and autonomous mobile robots in an environment cluttered with obstacles. Path planning is an important problem in the navigation of autonomous mobile robots. There are many path planning algorithms with obstacle avoidance, such as potential field [1-4], visibility graphs [5-6], grid methods [7], Lee-Algorithm [8-9], and virtual window [10].

Autonomous systems [11] allow a vehicle to move without any need for human control. These vehicles are also called driverless car or self-driving car. Advanced control systems interpret sensory information to identify appropriate navigation paths as well as obstacles and relevant sinage [12-13].

Potential field method assumes that all entities in the environment generate an artificial field around themselves in such a way that a mobile robot is attracted to its goal or target, while at the same time is repulsed by obstacles. The potential field approach can be used as a global motion planning algorithm.

The visibility graph method constructs a graph of vertices of polygons representing obstacles. It means that two vertices are connected in the graph if they are mutually visible. Lee's algorithm is a path finding algorithm and is normally applied for the placement of circuits on the printed circuit board. It guarantees to find a path between two points if it exists. This work concentrates on determining the path of a uniformly moving obstacle using two virtual windows. Once the path is determined, the mobile robot can then decides the next step in its action to avoid colliding with the obstacle.

A description of a virtual window is given along with its implementation within the context of this work. Then, the technique that is applied using two virtual windows to calculate the path of the uniformly 
moving obstacle is presented. A simulation applying this technique is performed to corroborate the effectiveness of the proposed system.

\section{METHODOLOGY}

\subsection{Discussion of the Virtual Window}

A virtual window is basically a rectangular plane that is projected ahead of a mobile robot for the purpose of detecting obstacles. In a vision system, an image of the view forward of the mobile robot is captured. This contains information of not only the plane of interest, but also of anything before and after that plane. The image maybe sharp at the plane of interest and blur in the vicinity of the plane of interest. A virtual window captures image just of the plane of interest. Information before and after the plane is disregarded. A full and complete discussion of the virtual window and its implementation is in [14]. The intersection of this virtual window with an obstacle will provide the mobile robot with the position of the obstacle. With this information, the mobile robot can then decide on the appropriate step to avoid collision with the obstacle.

Consider a single virtual window having a size of $1 \mathrm{~m} \times 1 \mathrm{~m}$ and is placed $1 \mathrm{~m}$ away from the mobile robot. As in a digital display or camera, a display resolution can be associated with this virtual window. This resolution is very much similar in concept to the pixels in a charge-coupled device chip.

The size (length and height of the virtual window) and pixel resolution can be set to any value that is required. Ideally, the size is usually rendered a little bigger than the size of the mobile robot. This allows for a bigger forward image to be monitored and thus a greater amount of information available for processing. This will give better protection to the mobile robot from collision with a moving obstacle compared to a similar or smaller size virtual window.

Let's assumed that the width of an obstacle in this scenario is not less than $0.1 \mathrm{~m}$, thus in order not to miss detecting the intersection with the virtual window, the recommended lowest resolution of the virtual window must be at least $10 \times 10$ pixels Figure 1 . Assuming the speed of light in air to be $2 \times 108 \mathrm{~ms}-1$, then the time taken for a single laser beam to a pixel on the virtual window and back to the sensor is about 10 nsecs. For a total pixel count of 100 , the total time is $1 \mu$ secs.

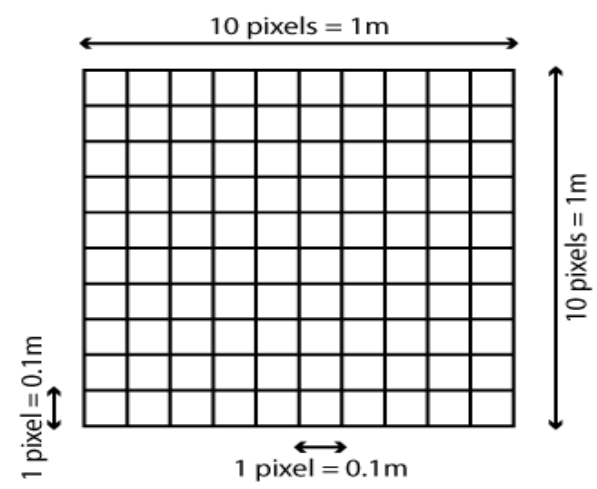

Figure 1. A visual image representation of the virtual window

For smaller obstacle sizes, higher resolution is recommended, though this will impact the processing time. However, for all practical purposes, current mobile robots are much bigger than 0.1m [15-17].

As another example, consider an obstacle having a width of $0.01 \mathrm{~m}$. The recommended lowest resolution for the virtual window is then at least $100 \times 100$ pixels. For this total pixel count of 10,000 , the total time taken for the scanning of the whole virtual window is $100 \mu$ secs or $0.1 \mathrm{~ms}$.

\subsection{Discussion Of The Implementation Of Two Virtual Windows}

The aim of this research work is to determine the path of the uniformly moving obstacle. For any path to be determined there must be at least two points of intersection. Since the obstacle is a uniformly moving object (having a straight path with constant speed), then obviously there must be two virtual windows placed forward of each other in order for the two intersections to occur Figure 2. Intersections with the two virtual windows will give sufficient information to calculate the path of the uniformly moving obstacle. 


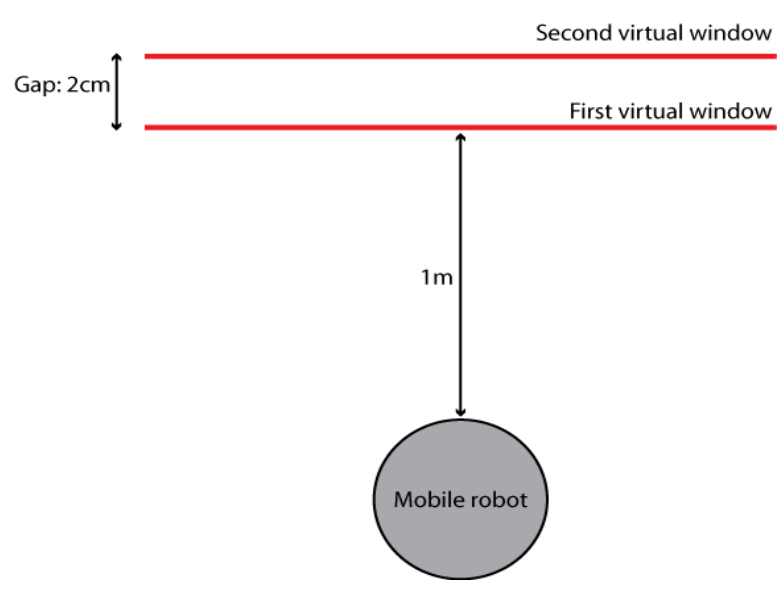

Figure 2. Top view showing the two virtual windows with respect to the mobile robot

Table 1 shows the relationship between the maximum theoretical speed of the moving obstacle that can still be detected with respect to the pixel resolution and gap between the two virtual windows. For a gap of $2 \mathrm{~cm}$ and with a pixel resolution of $10 \times 10$, the maximum theoretical speed that the moving obstacle can move while still being detected by the virtual windows is about $10,000 \mathrm{~ms}-1$ (equivalent to $36,000 \mathrm{kph}$, or $22,370 \mathrm{mph}$ ). This does not mean that the system cannot still determine the path at a higher speed, but there will be an associated offset error with speed greater than the theoretical maximum speed. This will be shown later in a simulation. For a more typical speed of an experimental mobile robot [18], the system is very much able to detect the intersections with the two virtual windows with ample time for processing.

Similarly, the maximum theoretical speed for a gap of $2 \mathrm{~cm}$ and pixel resolution of $100 \times 100$ is a more realistic $10 \mathrm{~ms}-1$. Maximum theoretical speeds for other values of the two parameters are shown in Table 1.

Table 1. Relationship Between Maximum Speed of Obstacle to the Pixel Resolution and Gap Between the Virtual Windows

\begin{tabular}{ccc}
\hline Gap bet. virtual windows & \multicolumn{2}{c}{ Pixel resolution } \\
\cline { 2 - 3 }$(\mathrm{cm})$ & $10 \times 10$ & $100 \times 100$ \\
2 & $10,000 \mathrm{~ms}^{-1}(\approx 36,000 \mathrm{kph})$ & $10 \mathrm{~ms}^{-1}(\approx 36 \mathrm{kph})$ \\
5 & $25,000 \mathrm{~ms}^{-1}$ & $250 \mathrm{~ms}^{-1}$ \\
10 & $50,000 \mathrm{~ms}^{-1}$ & $500 \mathrm{~ms}^{-1}$ \\
\hline
\end{tabular}

\section{SIMULATION RESULTS AND ANALYSIS}

\subsection{Determining the Path of the Obstacle With Respect to the Step Time of the Simulation}

For this simulation using Microsoft Excel ${ }^{\mathrm{TM}}$, the nearest virtual window (first virtual window) to the mobile robot is set at $1 \mathrm{~m}$ (or $100 \mathrm{~cm}$ ), while the second virtual window is located a further $0.02 \mathrm{~m}$ (or $2 \mathrm{~cm}$ ) from the first virtual window Figure 3. The start position of the obstacle is $(110,112)$ and the end point is $(2,4)$. The obstacle moves in a straight line as shown by the red dashes. The resolution of the virtual windows is $100 \times 100$ pixels and it is located $100 \mathrm{~cm}$ from the mobile robot, thus the time it takes to completely scan the two virtual windows is about $0.2 \mathrm{~ms}(0.0002 \mathrm{~s})$. The relative speed of the obstacle is assumed to be $5 \mathrm{~ms}-1$ (or $500 \mathrm{cms}-1$ ).

It is obvious from Figure 3 that the straight-line equation of the path of the obstacle is:

$$
\mathrm{y}=\mathrm{x}+2
$$

This equation will be used to verify the result from the simulation. The path of the moving obstacle will be calculated using the points of intersection with the two virtual windows.

The simulation is basically the calculation of the position of the moving obstacle every $0.0002 \mathrm{~s}$. A sample of the calculations for the positions of the obstacle every $0.0002 \mathrm{~s}$ is shown in Figure 4 . For this calculation, the speed of the obstacle is given as $500 \mathrm{cms}-1$ (or $5 \mathrm{~ms}-1$, which is half of the theoretical maximum speed as shown in Table 1), thus having component speeds for both $x$ and $y$ direction as $353.5534 \mathrm{cms}-1$. 


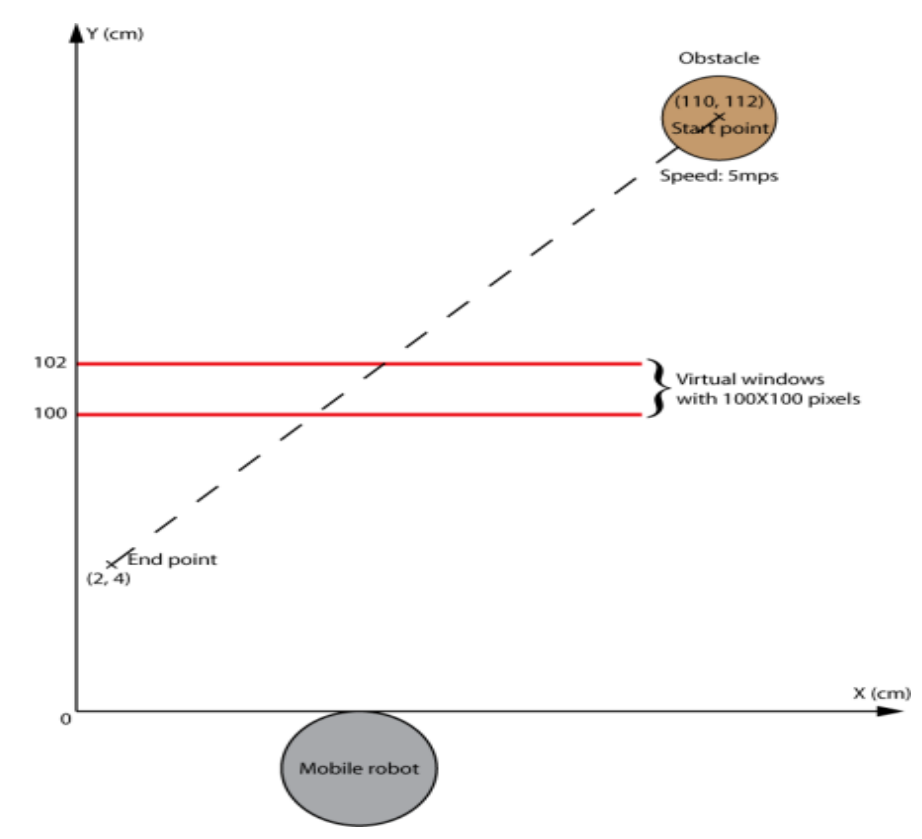

Figure 3. Top view showing relative position of the mobile robot and the obstacle, along with other relevant parameters

From Table 2, for the second virtual window, the intersection is only detected when the location of the obstacle is at $(99.4,101.4)$. The intersection coordinates with the first virtual window is at $(98,100)$. The coordinate $(100.1,102.1)$ is not valid because the obstacle has not intersect the second virtual window yet.

Using 1 for the path of the moving obstacle, conventional calculation gives the intersections as (100, $102)$ with the second virtual window, and $(98,100)$ with the first virtual window. The offset percentage error for the coordinates at the second virtual window is around $0.04 \%$, while the percentage error at the first is around $0.02 \%$.

\begin{tabular}{|c|c|c|}
\hline Time (second) & $\mathbf{x}$ & $\mathbf{y}$ \\
\hline $\mathbf{0 . 0 0 0 2}$ & 110 & 112 \\
\hline & 353.5534 & 353.5534 \\
\hline Time (second) & $\boldsymbol{x} \mathbf{~ c m}$ & $\boldsymbol{y} \mathbf{~ c m}$ \\
\hline 0 & 110.0 & 112.0 \\
\hline 0.000200 & 109.9 & 111.9 \\
\hline 0.000400 & 109.9 & 111.9 \\
\hline 0.000600 & 109.8 & 111.8 \\
\hline 0.000800 & 109.7 & 111.7 \\
\hline 0.001000 & 109.6 & 111.6 \\
\hline 0.001200 & 109.6 & 111.6 \\
\hline 0.001400 & 109.5 & 111.5 \\
\hline 0.001600 & 109.4 & 111.4 \\
\hline 0.001800 & 109.4 & 111.4 \\
\hline 0.002000 & 109.3 & 111.3 \\
\hline 0.002200 & 109.2 & 111.2 \\
\hline 0.002400 & 109.2 & 111.2 \\
\hline 0.002600 & 109.1 & 111.1 \\
\hline 0.002800 & 109.0 & 111.0 \\
\hline 0.003000 & 108.9 & 110.9 \\
\hline 0.003200 & 108.9 & 110.9 \\
\hline 0.003400 & 108.8 & 110.8 \\
\hline 0.003600 & 108.7 & 110.7 \\
\hline 0.003800 & 108.7 & 110.7 \\
\hline 0.004000 & 108.6 & 110.6 \\
\hline 0.004200 & 108.5 & 110.5 \\
\hline 0.004400 & 108.4 & 110.4 \\
\hline 0.004600 & 108.4 & 110.4 \\
\hline & & \\
\hline
\end{tabular}

(a)

\begin{tabular}{|c|c|c|}
\hline 0.028200 & 100.03 & 102.03 \\
\hline 0.028400 & 99.96 & 101.96 \\
\hline 0.028600 & 99.89 & 101.89 \\
\hline 0.028800 & 99.82 & 101.82 \\
\hline 0.029000 & 99.75 & 101.75 \\
\hline 0.029200 & 99.68 & 101.68 \\
\hline 0.029400 & 99.61 & 101.61 \\
\hline 0.029600 & 99.53 & 101.53 \\
\hline 0.029800 & 99.46 & 101.46 \\
\hline 0.030000 & 99.39 & 101.39 \\
\hline 0.030200 & 99.32 & 101.32 \\
\hline 0.030400 & 99.25 & 101.25 \\
\hline 0.030600 & 99.18 & 101.18 \\
\hline 0.030800 & 99.11 & 101.11 \\
\hline 0.031000 & 99.04 & 101.04 \\
\hline 0.031200 & 98.97 & 100.97 \\
\hline 0.031400 & 98.90 & 100.90 \\
\hline 0.031600 & 98.83 & 100.83 \\
\hline 0.031800 & 98.76 & 100.76 \\
\hline 0.032000 & 98.69 & 100.69 \\
\hline 0.032200 & 98.62 & 100.62 \\
\hline 0.032400 & 98.54 & 100.54 \\
\hline 0.032600 & 98.47 & 100.47 \\
\hline 0.032800 & 98.40 & 100.40 \\
\hline 0.033000 & 98.33 & 100.33 \\
\hline 0.033200 & 98.26 & 100.26 \\
\hline 0.033400 & 98.19 & 100.19 \\
\hline 0.033600 & 98.12 & 100.12 \\
\hline 0.033800 & 98.05 & 100.05 \\
\hline 0.034000 & 97.98 & 99.98 \\
\hline 0.034200 & 97.9 & 99.9 \\
\hline & $(b)$ & \\
\hline
\end{tabular}

(b)

Figure 4. (a) A sample of the calculations as an image captured from Microsoft Excel ${ }^{\mathrm{TM}}$ at a time step of $0.0002 \mathrm{~s}$, (b) A sample of the results with the two intersections highlighted and high precision simulated values 
Table 2. The Position Coordinates of the Moving Obstacle in the Vicinity of First $(\mathrm{y}=100 \mathrm{~cm})$ and Second $(\mathrm{y}=102 \mathrm{~cm})$ Virtual Windows

\begin{tabular}{ccc}
\hline \multirow{2}{*}{ Virtual window } & \multicolumn{2}{c}{ Coordinates $(\mathrm{cm})$} \\
\cline { 2 - 3 } Second & $x$ & $y$ \\
& 100.03 & 102.03 \\
First & 99.96 & 101.96 \\
& 98.05 & 100.05 \\
& 97.98 & 99.98 \\
\hline
\end{tabular}

From the coordinates of intersection in Table 2, the equation of the path of the obstacle is found to be:

$$
y=x+2
$$

The path of the obstacle obtained through the simulation is exactly the same as the set path of the obstacle 1. Figure 5 shows the path of the obstacle achieved through simulation and conventional calculation. The reason for the offset is that the system is assumed to detect the intersection with the second virtual window at $y=101.96$, instead of 102 . This is because the calculation was done in steps of $0.0002 \mathrm{~s}$, corresponding to the time taken for the system to scan the two virtual windows. The error as shown above is very small, less than $0.05 \%$.

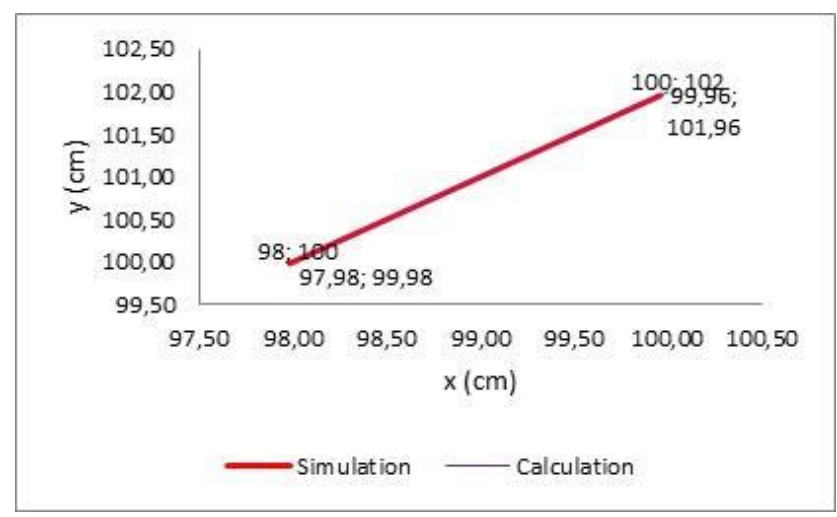

Figure 5. Comparison of the simulated path against the calculated path of the moving obstacle for a time step of $0.0002 \mathrm{~s}$

Alternatively, the coordinates for the two intersections with both virtual windows can be considered to be $(97.98,100)$ instead of $(97.98,99.98)$ for the first virtual window, and $(99.96,102)$ instead of $(99.96$, 101.96) for the second. These coordinates can be accepted if the system presumed that the intersections happened at $\mathrm{y}=100$ for the first virtual window, and $\mathrm{y}=102$ for the second virtual window even though it was detected a little bit further than the actual position of the virtual windows.

Figure 6 shows the simulated and calculated path of the moving obstacle. As can be seen, the slopes are almost similar. The slope for the simulation is found to be 1.01, thus giving a percentage error of $1 \%$. The y-intercept, however, is found to be 1.04 and this gives a percentage error of about $48 \%$. Even though the error of the y-intercept is large, this can be disregarded because it depends on the positioning of the axes. If the axes were placed nearer to the centre of the mobile robot, then the error is expected to be smaller, i.e. the y-intercept will be nearer to the calculated y-intercept of 2 . In this study, the slope is more relevant than the y-intercept. The percentage error for the slope is just $1 \%$. 


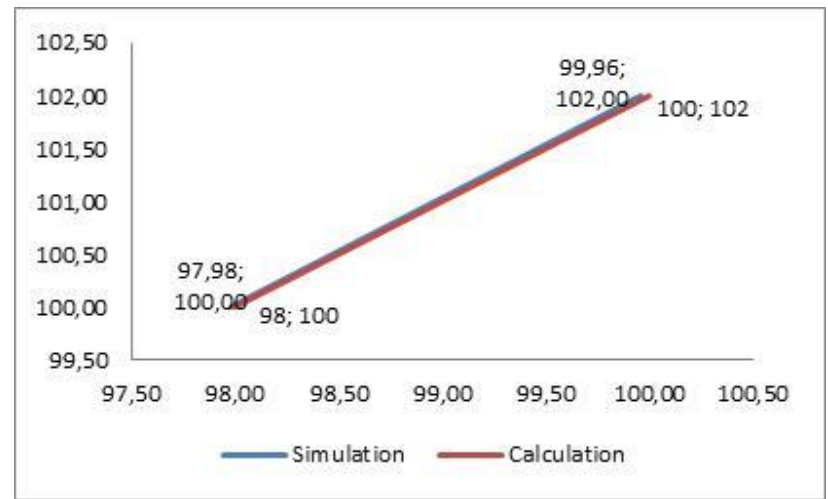

Figure 6. Comparison of the simulated path against the calculated path of the moving obstacle for a time step of $0.0002 \mathrm{~s}$ and taking the y intersections as being 102 and 100 for the second and first virtual window respectively

Figure 7 shows another graph with the moving obstacle having a higher speed at $1000 \mathrm{cms}-1$ (10ms-1). This is twice the speed of the previous exercise and at the limit of this virtual window (Table 1). The percentage errors for the slope and the y-intercept are the same as the previous exercise. This can be seen from the graph where the intersections are the same as in the previous exercise.

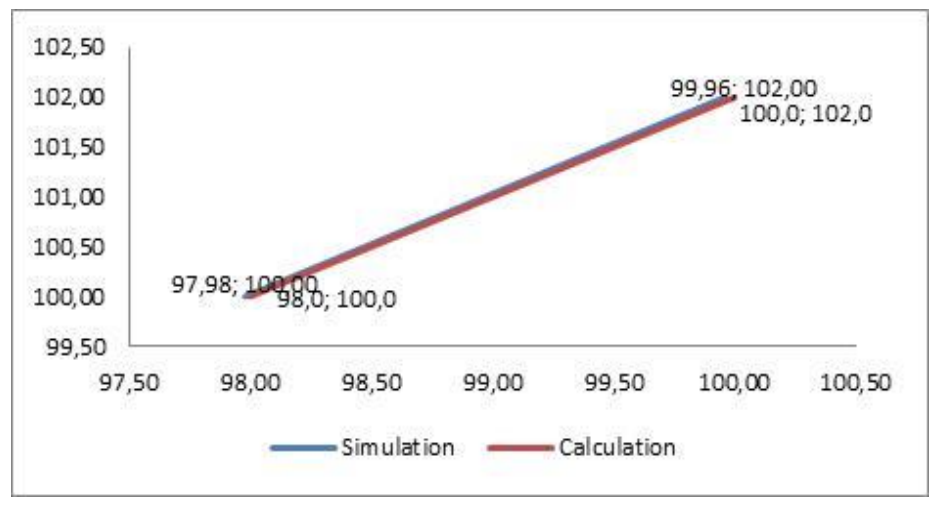

Figure 7. Comparison of the simulated path against the calculated path of the moving obstacle for a time step of $0.0002 \mathrm{~s}$ and with obstacle speed of $10 \mathrm{~ms}-1$ and taking the $y$ intersections as being 102 and 100 for the second and first virtual window respectively

Another example is performed with a higher speed of $10 \mathrm{~ms}-1$ (1000 cms-1). Table 3 shows the point of intersections. It is the same as the obstacle with a speed of $500 \mathrm{cms}-1$, and thus the percentage errors are similar too. On the other hand, if the speed is increased to $1200 \mathrm{cms}-1$, the slope percentage error increased to $2 \%$, while similarly the $y$-intercept percentage error is calculated to be $100 \%$. This corroborates the limits that was calculated and shown in Table 1.

Table 3. The Position Coordinates of the Moving Obstacle in the Vicinity of First $(\mathrm{y}=100 \mathrm{~cm})$ and Second $(y=102 \mathrm{~cm})$ Virtual Windows Having a Speed of $1000 \mathrm{cms}-1$

\begin{tabular}{ccc}
\hline \multirow{2}{*}{ Virtual window } & \multicolumn{2}{c}{ Coordinates $(\mathrm{cm})$} \\
\cline { 2 - 3 } Second & $x$ & $y$ \\
& 99.96 & 101.96 \\
First & & $(102.0)$ \\
& 97.98 & 99.98 \\
& & $(100.0)$ \\
\hline
\end{tabular}


For comparison, another example is given with a time step of $0.005 \mathrm{~s}$. Figure 8 show a sample of the output from the simulation and Figure 9 the path plotted as a graph.

\begin{tabular}{|c|c|c|}
\hline Time (second) & $\mathbf{x}$ & $\mathbf{y}$ \\
\hline $\mathbf{0 . 0 0 5}$ & 110 & 112 \\
\hline & 353.5534 & 353.5534 \\
\hline Time (second) & $\boldsymbol{x} \mathbf{~ c m}$ & $\boldsymbol{y} \mathbf{~ c m}$ \\
\hline 0 & 110.0 & 112.0 \\
\hline 0.005000 & 108.2 & 110.2 \\
\hline 0.010000 & 106.5 & 108.5 \\
\hline 0.015000 & 104.7 & 106.7 \\
\hline 0.020000 & 102.9 & 104.9 \\
\hline 0.025000 & 101.2 & 103.2 \\
\hline 0.030000 & 99.39 & 101.39 \\
\hline 0.035000 & 97.63 & 99.63 \\
\hline 0.040000 & 95.9 & 97.9 \\
\hline 0.045000 & 94.1 & 96.1 \\
\hline 0.050000 & 92.3 & 94.3 \\
\hline 0.055000 & 90.6 & 92.6 \\
\hline 0.060000 & 88.8 & 90.8 \\
\hline 0.065000 & 87.0 & 89.0 \\
\hline 0.070000 & 85.3 & 87.3 \\
\hline 0.075000 & 83.5 & 85.5 \\
\hline 0.080000 & 81.7 & 83.7 \\
\hline
\end{tabular}

Figure 8. A sample of the calculations as an image captured from Microsoft Excel ${ }^{\mathrm{TM}}$ at a time step of $0.005 \mathrm{~s}$

The slope was found to be 1.14 giving a percentage error of $14 \%$. The y-intercept is at -11.31 . This gives a percentage error of $665.4 \%$. These values corroborates the limits that was given in Table 1 .

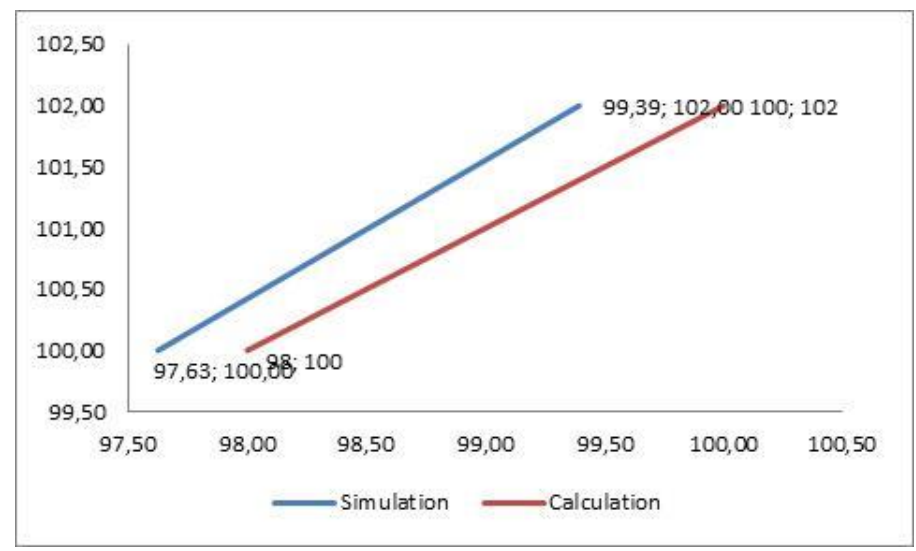

Figure 9. Comparison of the simulated path against the calculated path of the moving obstacle for a time step of $0.005 \mathrm{~s}$

\section{CONCLUSION}

It was shown that the system was able to determine the path of the moving obstacle so long as there is an intersection each with the two virtual windows. For the exercises did, the percentage offset error was found to be less than $0.1 \%$.

This bodes very well for the system as the offset error does not affect the correct determination of the path of the uniformly moving obstacle.

\section{ACKNOWLEDGMENT}

The authors would like to acknowledge that this research project is funded from a grant awarded by the Ministry of Higher Education: FRGS/1/2017/ICT04/UITM/02/5. 


\section{REFERENCES}

[1] Ge SS \& Cui YJ, "Dynamic motion planning for mobile robots using potential field method", Proc. IEEE Int. Conf. Autonomous Robots, vol. 13(3), pp. 207 - 222, 2002.

[2] Yin L et al, "A new potential field method for mobile robot path planning in the dynamic environments", Asian Journal of Control, vol. 11(2), pp. 214 - 225, 2009.

[3] Sugiyama S et al, "Path planning of a mobile robot for avoiding moving obstacles with improved velocity control by using the hydrodynamic potential", IEEE Int. Conf. on Intelligent Robots and Systems, 2010.

[4] Liu C et al, "Path planning of mobile robot using new potential field method in dynamic environment", IEEE Int. Conf. on Natural Computation, 2011.

[5] Li L et al, "Present state and future development of mobile robot technology research", Robot, vol. 24(5), pp. 475 480, 2002.

[6] Raja P \& Pugazhenti S, "Path planning for a mobile robot in dynamic environments", Int. Journal of Physical Sciences, vol. 6(20), pp. $4721-4731,2011$.

[7] Boschian V \& Pruski A, "Grid modeling of robot cells: A memory-efficient approach", Journal of Intelligent and Robotic Systems, vol. 8(2), pp. 201 - 203,1993.

[8] Maciej P et al, "Lee-algorithm based path replanner for dynamic environments", Int. Conf. on Signals and Electronic Systems, 2012.

[9] Maciej P et al, "Obstacle avoidance procedure and Lee algorithm based path replanner for autonomous mobile platforms", Int. Journal of Electronics and Telecommunications, vol. 59(1), pp. 85 - 91, 2013.

[10] Mansor MA \& Morris AS, "Path planning in unknown environment with obstacles using virtual window", Journal of Intelligent and Robotic Systems, vol. 24(3), pp. 235 - 251, 1999.

[11] Thrun, Sebastian, "Toward Robotic Cars". Communications of the ACM. vol. 53 (4) pp 99-106, 2010.

[12] Lassa, Todd "The Beginning of the End of Driving". Motor Trend. (January 2013). Retrieved 1 September 2014.

[13] European Roadmap Smart Systems for Automated Driving, European Technology Platform on Smart Systems Integration (EPoSS), 2015.

[14] Mansor MA, "Modelling and Simulation of Mobile Robot Manipulators Moving in a Space with Obstacle", PhD Thesis, University of Sheffield, May 1998.

[15] http://www.robotshop.com/4wd-omni-directional-mobile-robot-kit-2.html

[16] http://www.dfrobot.com/index.php?route=product/product\&path=37\&product_id=361

[17] http://www.coreconagvs.com/products/

[18] Chung W et al, "High speed navigation of a mobile robot based on robot's experiences", JSME Robotics and Mechatronics Conference, 2006. 